\title{
Methanolic extract of Momordica cymbalaria enhances glucose uptake in L6 myotubes in vitro by up-regulating PPAR- $\gamma$ and GLUT-4
}

\author{
Puttanarasaiah Mahesh Kumar ${ }^{1 *}$, Marikunte V Venkataranganna ${ }^{2}$, Kirangadur Manjunath ${ }^{3}$, \\ Gollapalle L Viswanatha ${ }^{4}$, Godavarthi Ashok ${ }^{5}$ \\ ${ }^{I}$ Padmashree Institute of Information Sciences, Bangalore 560072, India; \\ ${ }^{2}$ Connexios Life Sciences Pvt Ltd., J.P.Nagar, Bangalore 560078, India; \\ ${ }^{3}$ Department of Microbiology and Biotechnology, Bangalore University, Bangalore 560056, India; \\ ${ }^{4}$ PES College of Pharmacy, Bangalore 560050, India; \\ ${ }^{5}$ Director, Radiant Research Services Pvt Ltd., Bangalore 560050, India
}

Available online 20 Dec. 2014

\begin{abstract}
[ABSTRACT] The present study was undertaken to evaluate the influence of the methanolic fruit extract of Momordica cymbalaria (MFMC) on PPAR $\gamma$ (Peroxisome Proliferator Activated Receptor gamma) and GLUT-4 (Glucose transporter-4) with respect to glucose transport. Various concentrations of MFMC ranging from 62.5 to $500 \mu \mathrm{g} \cdot \mathrm{mL}^{-1}$ were evaluated for glucose uptake activity in vitro using L6 myotubes, rosiglitazone was used as a reference standard. The MFMC showed significant and dose-dependent increase in glucose uptake at the tested concentrations, further, the glucose uptake activity of MFMC $\left(500 \mu \mathrm{g} \cdot \mathrm{mL}^{-1}\right)$ was comparable with rosigilitazone. Furthermore, MFMC has shown up-regulation of GLUT-4 and PPAR $\gamma$ gene expressions in L6 myotubes. In addition, the MFMC when incubated along with cycloheximide (CHX), which is a protein synthesis inhibitor, has shown complete blockade of glucose uptake. This indicates that new protein synthesis is required for increased GLUT-4 translocation. In conclusion, these findings suggest that MFMC is enhancing the glucose uptake significantly and dose dependently through the enhanced expression of PPAR $\gamma$ and GLUT-4 in vitro.
\end{abstract}

[KEY WORDS] Momordica cymbalaria; Diabetes; Glucose transport; PPAR $\gamma$; L6 myotubes; Antidiabetic activity

[CLC Number] R965 [Document code] A [Article ID] 2095-6975(2014)12-0895-06

\section{Introduction}

Medicinal plants with their natural phytoconstituents form a base for the discovery of new chemical entities in modern drug discovery. A significant number of plant-derived active principles having therapeutic use in the treatment of type 2 diabetes mellitus have been reported ${ }^{[1]}$. In this context, the various plant parts of Momordica cymbalaria Fenzl ex

[Received on] 02-Feb.-2014

[Research funding] The project was supported by Padmashree Institute of Information Sciences, Bangalore.

[*Corresponding author] P. Mahesh Kumar: Tel: +91-9844492334, +91-80-28485445, Fax: +91-80-28485445, E-mail: maheshguni (a)yahoo.com

These authors have no any conflict of interest to declare.

Published by Elsevier B.V. All rights reserved
Naudin (Cucurbitaceae) have been demonstrated to have antidiabetic and hypoglycemic activity ${ }^{[2-4]}$. However, the rate limiting step in glucose utilization is glucose transport, especially in insulin-targeted skeletal muscle, mediated by major glucose transporters (GLUT proteins, GLUT-4 and GLUT-1) ${ }^{[5]}$. Cloning and characterization of insulin responsive glucose transporters has revealed that GLUT-1 and GLUT-4 are expressed in human skeletal muscle ${ }^{[6]}$. Insulin resistance in type 2 diabetes is characterized by decreased insulin-stimulated glucose transport and weakened metabolism in adipocytes and skeletal muscle resulting in down-regulation of the major insulin responsive GLUT's ${ }^{[7]}$.

Weakened GLUT-4 translocation, and reduced expression of peroxisome proliferator activated receptor gamma (PPAR $\gamma$ ) have been studied under diabetic conditions. Studies have shown the role of PPAR $\gamma$ in insulin signaling, and also enumer- 
ated the up-regulation of insulin-dependent glucose transport and GLUT-4 translocation in cultured L6 myotubes ${ }^{[8]}$.

$\operatorname{PPAR} \gamma$, a transcription factor belonging to the nuclear receptor superfamily ${ }^{[9]}$, is essential for adipocyte differentiation ${ }^{[10]}$, and PPAR $\gamma$ agonists directly enhances insulin signalling and glucose uptake in the muscles by binding with the PPAR $\gamma$ receptors ${ }^{[8]}$.

Previously, various plant parts of $M$. cymbalaria, were extensively studied for their hypoglycemic and antidiabetic activity. However, no studies related to its mechanism of action have been carried out, and hence the molecular aspects of the mechanism behind the hypoglycemic and antidiabetic effects of M. cymbalaria need to be explored. With this background, the present study was carried out to exemplify the molecular mechanistic action of MFMC, at the cellular level, by evaluating the influence of MFMC on glucose transport (GLUT-4), and PPAR $\gamma$ expression using L6 myotubes in vitro.

\section{Materials and Methods}

Reagents, chemicals, and cell lines

All cell culture supplements were purchased from Sigma-Aldrich (St. Louis, MO, USA). All fine chemicals, TRI reagent, and Avian Moloney Leukemic virus reverse transcriptase (AMLV), dNTP, and Taq polymerase were also purchased from Sigma-Aldrich. All other reagents and chemicals purchased were of molecular biology grade and were purchased from Sigma-Aldrich.

Collection of plant material

The fruits of Momordica cymbalaria, were collected from Hospete (Bellary District, Karnataka, India), in the period of May-June 2012, considered the seasonal conditions for obtaining maximum phytoconstituents. The collected plant material was authentified and certified by Prof. K. P. Srinath, Department of Botany, Bangalore University, Bangalore, a voucher specimen of the plant material is preserved in the department with specimen no. 2012-13/MC/BT-01.

Plant material preparation

The fruits of Momordica cymbalaria, were shade-dried and powdered. The fruit powder $(50 \mathrm{~g})$ was extracted sequentially with hexane, dichloromethane, ethyl acetate, and methanol, in a Soxhlet apparatus. Initially, the plant material was extracted with hexane $(1 \mathrm{~L})$ at $60{ }^{\circ} \mathrm{C}$ for $24 \mathrm{~h}$, the marc obtained was completely dried, and extracted with dichloromethane $(1 \mathrm{~L})$ for $24 \mathrm{~h}$, and then the marc was extracted with ethyl acetate $(1 \mathrm{~L})$, followed by methanol $(1 \mathrm{~L})$.

Extracts were concentrated in a rotor evaporator (Remi Instruments), under reduced pressure at room temperature, and dried extract $(1 \mathrm{mg})$ was reconstituted to $1 \mathrm{~mL}$ with respective solvents, and diluted to attain a final concentration of $500 \mu \mathrm{g} \cdot \mathrm{mL}^{-1}$, from the stock solution of $500 \mu \mathrm{g} \cdot \mathrm{mL}^{-1}$ further concentrations were prepared by serial dilution $(250,125$, $62.5 \mu \mathrm{g} \cdot \mathrm{mL}^{-1}$ ), for glucose uptake studies.

Propagation and maintenance of L6 cells

L6 cell cultures (rat skeletal muscle), was procured from the National Centre for Cell Sciences (NCCS), Pune, India. L6 cells were cultured and maintained in Dulbecco's modified Eagle's medium (DMEM), with 10\% inactivated Fetal Bovine serum (FBS), along with penicillin $\left(100 \mathrm{IU} \cdot \mathrm{mL}^{-1}\right)$, streptomycin $\left(100 \mu \mathrm{g} \cdot \mathrm{mL}^{-1}\right)$, and amphotericin $\mathrm{B}\left(5 \mu \mathrm{g} \cdot \mathrm{mL}^{-1}\right)$, in a humidified atmosphere of $5 \% \mathrm{CO}_{2}$ at $37{ }^{\circ} \mathrm{C}$, until confluence. The cells were dissociated with TPVG solution ( $0.2 \%$ trypsin, $0.02 \%$ EDTA, and $0.05 \%$ glucose in PBS). The stock cultures were grown in $25 \mathrm{~cm}^{2}$ culture flasks.

Glucose uptake assay

Completely differentiated myotubes demonstrates a high response to insulin, and rosiglitazone mediated glucose uptake. The differentiated myotubes were serum-starved overnight, and washed with HEPES in Kreb's Ringer phosphate solution (KRP buffer), and incubated with KRP buffer with $0.1 \%$ BSA (Bovine serum albumin) for $30 \mathrm{~min}$ at $37{ }^{\circ} \mathrm{C}$. The myotubes were treated with various concentrations of plant extract, and standard along with vehicle control, in $60 \mathrm{~mm}$ Petri plates. Subsequently, D-glucose solution was added to all the plates, and incubated for $30 \mathrm{~min}$ at $37{ }^{\circ} \mathrm{C}$. Subsequently, the liquid medium was aspirated from all the plates, to terminate the glucose uptake, and the cells were washed three times with ice-cold KRP buffer solution. Further, the cells were lysed with $0.1 \mathrm{~mol} \cdot \mathrm{L}^{-1} \mathrm{NaOH}$ solution, and an aliquot of the cell lysate was used to measure the cell-associated glucose. Glucose uptake was estimated by using Biovision glucose estimation kit (Biovision Kit Inc, USA). Three independent experimental values, in duplicates were taken to determine the percentage enhancement of glucose uptake compared with control ${ }^{[11-12]}$.

Reverse transcriptase-polymerase chain reaction (RT-PCR)

RT-PCR was performed as published ${ }^{[11]}$. In short, after the completion of incubation, the cells were lysed in TRI Reagent, the protein content was extracted with chloroform, and the total RNA was precipitated with isopropanol. The RNA precipitate was washed with $70 \%$ ethanol, and resuspended in DEPC-treated water $(50 \mu \mathrm{L})$. Reverse transcription was performed using 200 units of avian reverse transcriptase, and $200 \mathrm{ng} \cdot \mu \mathrm{L}^{-1}$ oligo $\mathrm{d}(\mathrm{T}) 18$. The primers used were as follows, GLUT-4: sense, 5'-CGG GAC GTG GAG CTG GCC GAG GAG-3'; anti-sense, 5'CCC CCT CAG CGA GTG A-3' (318-bp) ${ }^{[12]}$; PPAR $\gamma$; sense, 5'-GGA TTC ATG ACC AGG GAG TTC CTC-3'; anti-sense, 5'-GCG GTC TCC ACT GAG

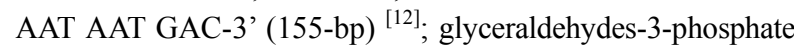
dehydrogenase (GAPDH); sense, 5'CCA CCC ATG GCA AAT TCC ATG GCA-3'; anti-sense, 5'-TCT AGA CGG CAG GTC AGG TCC ACC - 3' (588-bp) ${ }^{[12]}$. For PCR reaction, cDNA mixture $(1 \mu \mathrm{L})$ was added to a PCR reaction mix containing $10 \times$ PCR buffer, $2 \mathrm{mmol} \cdot \mathrm{L}^{-1} \mathrm{dNTP}, 10 \mathrm{pmol} \cdot \mathrm{L}^{-1}$ of paired primers, and 2 units of Taq polymerase. PCR products were run on $1.5 \%$ agarose gels, stained with ethidium bromide, and the gel was photographed by scanning densitometer.

Statistical analysis

All the values were expressed as $\bar{x} \pm$ SEM. The results 
were analyzed statistically using one way ANOVA followed by Tukey's multiple comparison test using Graph Pad Prism version 4.03 for Windows, Graph Pad Software, San Diego CA, USA. The minimum level of significance was fixed at $P<0.05$.

\section{Results}

\section{Extraction of the plant material}

The fruits powder of M. cymbalaria was successively extracted with hexane, dichloromethane, ethyl acetate, and methanol. The extractive values of hexane, dichloromethane, ethyl acetate, and methanol were found to be $0.6 \%, 0.45 \%$,
$2.3 \%$, and $3.2 \% \mathrm{~W} / \mathrm{W}$, respectively.

Evaluation of glucose uptake

The glucose uptake activity of MFMC was evaluated using a $50 \mu \mathrm{g} \cdot \mathrm{mL}^{-1}$ concentration of rosiglitazone as reference standard, based on a pilot study (unpublished data). The MFMC at concentrations ranging from 62.5 to $500 \mu \mathrm{g} \cdot \mathrm{mL}^{-1}$ showed dose-dependent stimulation of glucose uptake, at $500 \mu \mathrm{g} \cdot \mathrm{mL}^{-1}$ concentration it offered approximately two-fold increase in glucose uptake from the basal concentration of glucose, similarly rosiglitazone also showed a significant increase in glucose uptake. The results are given in Fig. 1.

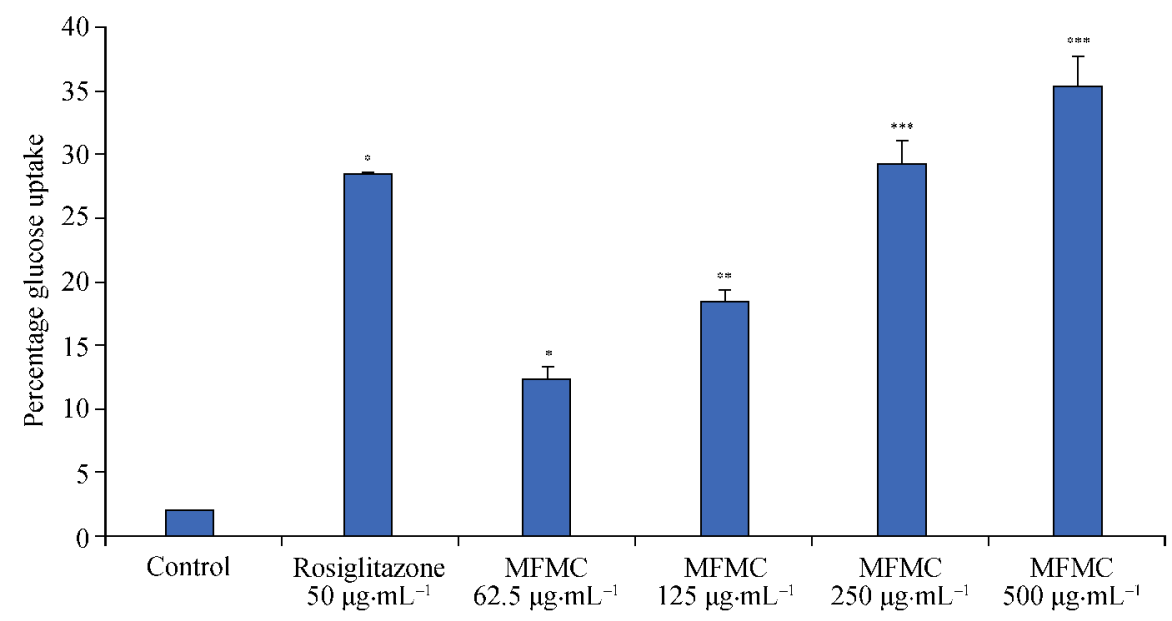

Fig. 1 Glucose uptake activity of methanolic extract of Momordica cymbalaria (MFMC) in L6 myotubes $(\bar{x} \pm \operatorname{SEM}, n=3$ ) Means of various groups were statistically compared by one way ANOVA followed by Tukey's multiple comparison test using Graph Pad version 4.03. ${ }^{\dagger} P<0.001$ corresponds to rosiglitazone vs control; ${ }^{*} P<0.05,{ }^{* *} P<0.01,{ }^{* * *} P<0.001$ vs MFMC vs control

Further, the effect of MFMC on glucose uptake was thought to be mediating through enhanced expression of GLUT-4, and PPAR $\gamma$ synthesis, and thereby enhanced protein synthesis. To confirm this hypothesis, another set of experiment was performed where the glucose uptake activity of MEMC was carried out in the presence of a protein synthesis inhibitor cycloheximide (CHX) in L6 myotubes, along with the previously included experimental groups. Remarkably, the glucose uptake activity of MFMC was abolished in the presence of CHX, which suggests that the enhanced glucose uptake activity of MFMC, is mediating through enhanced protein synthesis (Fig. 4).

Effect of MFMC on GLUT-4 and PPAR $\gamma$ transcription levels

The effect of MFMC on GLUT-4, and PPAR $\gamma$ mRNA expression was determined by semi-quantitative RT-PCR. The MFMC and rosiglitazone elevated the transcription of GLUT-4 in L6 myotubes during $24 \mathrm{~h}$ of incubation, when compared to control. The scanning densitometric analysis of the agarose gel showed approximately a 2.1-fold increase in GLUT-4 transcription levels in the MFMC $\left(500 \mu \mathrm{g} \cdot \mathrm{mL}^{-1}\right)$ treated group, when compared to control. The results are depicted in Figs. 2A, 2B, and 2C.

Additionally, the role of PPAR $\gamma$ expression in glucose transport mediated by MFMC was investigated. Surprisingly, the MFMC treatment showed approximately a 2.5 -fold increased expression of $\operatorname{PPAR} \gamma$, when compared to control. These findings were in agreement with the findings of the in vitro glucose uptake assay. Further, the L6 myotubes were analyzed for the GAPDH transcripts at $24 \mathrm{~h}$ (588bp). The representative signals of PPAR $\gamma$ in the agarose gel were quantified arbitrarily in three independent experiments, and representative agarose gels are shown Figs. 3A, 3B, and 3C.

All these findings were in agreement with the conclusion that the enhanced glucose uptake activity of MFMC is mediating through enhanced gene expression of GLUT-4 and PPAR- $\gamma$. Furthermore, the enhanced protein synthesis is the key factor in enhancing the glucose uptake, which was confirmed when the glucose uptake was completely abolished in presence of cycloheximide (CHD), which is a protein synthesis inhibitor (Fig. 4).

\section{Discussion}

The abnormal glucose transport associated with deficient GLUT-4 translocation, and/or faulty insulin signaling cascade are manifested as among the dominant defects in insulin resistance in type 2 diabetes. The intense rise in GLUT- 4 and 

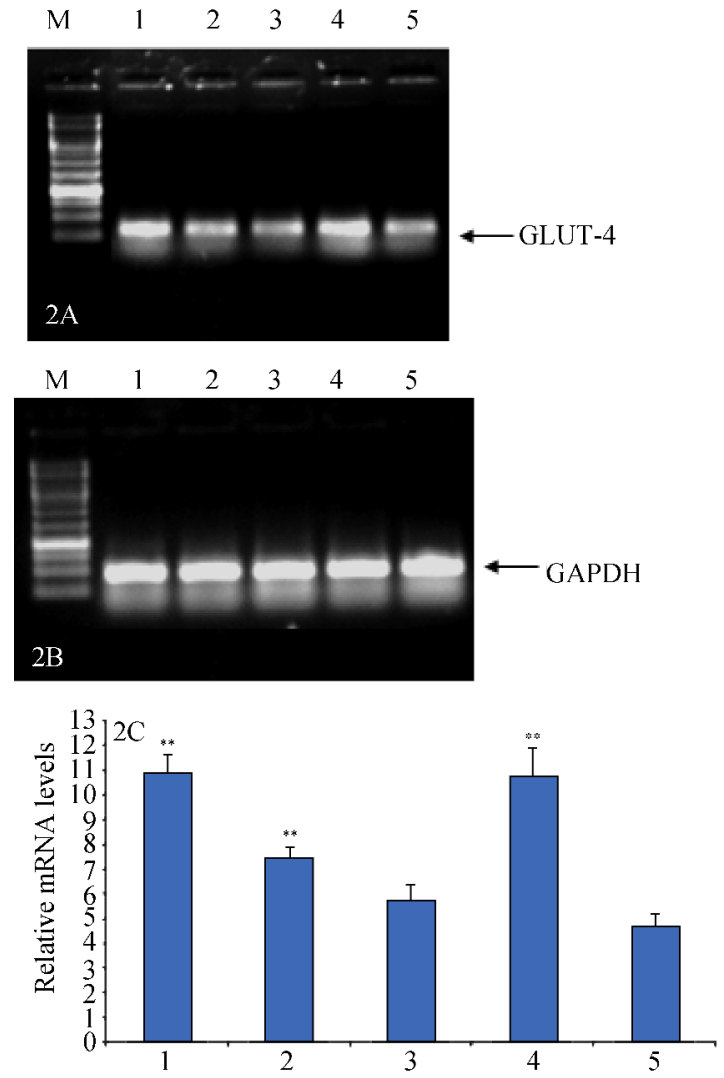

Fig. 2 Effect of the methanolic fruit extract of Momordica cymbalaria (MFMC) on Glut-4 transcripts in L6 myotubes $(\bar{x} \pm$ SEM, $n=3)$

M: Ikbp marker Lane 1: Indicates $500 \mu \mathrm{g} \cdot \mathrm{mL}^{-1} \mathrm{MFMC}$, Lane 2: indicates $250 \mu \mathrm{g} \cdot \mathrm{mL}^{-1} \mathrm{MFMC}$, Lane 3: $125 \mu \mathrm{g}$ MFMC, Lane 4: Rosiglitazone, Lane 5: control

Means of various groups were statistically compared by one way ANOVA followed by Tukey's multiple comparison test using Graph Pad version 4.03. ${ }^{*} P<0.05,{ }^{* * *} P<0.01$ vs control
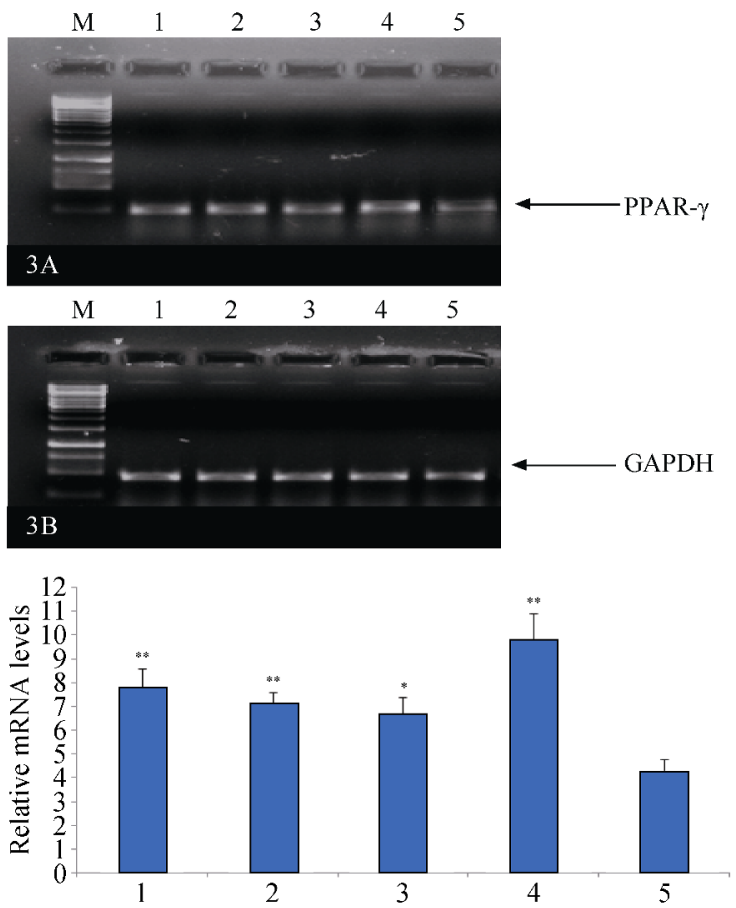

Fig. 3 Effect of methanolic fruit extract of Momordica cymbalaria (MFMC) on PPAR $\gamma$ transcripts in L6 myotubes $(\bar{x} \pm$ SEM, $n=3)$

M: Ikbp marker Lane 1: indicates $500 \mu \mathrm{g} \cdot \mathrm{mL}^{-1}$ MFMC, Lane 2: indicates $250 \mu \mathrm{g} \cdot \mathrm{mL}^{-1}$ MFMC, Lane 3: $125 \mu \mathrm{g}$ MFMC, Lane 4: Rosiglitazone, Lane 5: control

Means of various groups were statistically compared by one way ANOVA followed by Tukey's multiple comparison test using Graph Pad version 4.03. $P<0.05,{ }^{* *} P<0.01$ vs control

P13 kinase, mRNA levels in euglycemic and hyperinsulinemic clamp in the presence of insulin refined the role of GLUT-4, and P13 kinase in insulin-mediated glucose

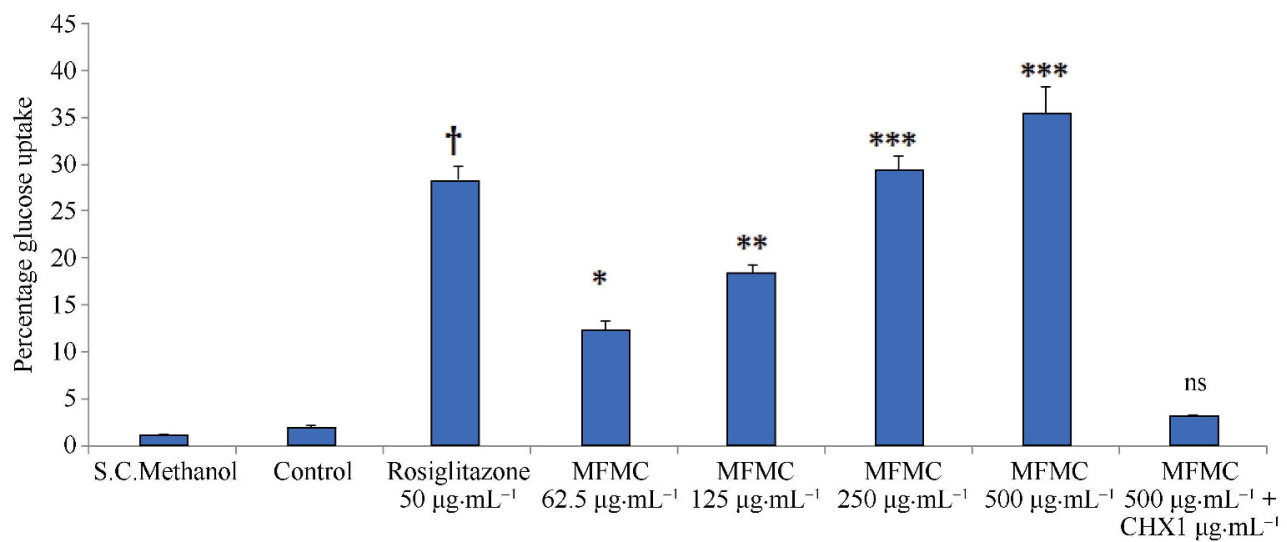

Fig. 4 Effect of cycloheximide on methanolic fruit extract of Momordica cymbalaria (MFMC) intervening glucose uptake.

Note : ns, Not significant $(\bar{x} \pm$ SEM, $n=3)$

Means of various groups were statistically compared by one way ANOVA followed by Tukey's multiple comparison test using Graph Pad version 4.03. ${ }^{\dagger} P<0.001$ corresponds to Rosiglitazone $v$ s control; ${ }^{*} P<0.05,{ }^{* * *} P<0.01,{ }^{* * * *} P<0.001$ corresponds to MFMC vs control. ${ }^{\text {ns } P}>$ 0.05 corresponds to MFMC $+\mathrm{CHX} v \mathrm{vs}$ MFMC $62.5 \mu \mathrm{g} \cdot \mathrm{mL}^{-1}$ 
transport ${ }^{[13]}$. Apart from insulin, PPAR $\gamma$ agonists like rosiglitazone and pioglitazone have been shown to facilitate glucose transport in type 2 diabetes by acting as insulin sensitizers ${ }^{[14]}$. Hence the up-regulation of such molecular switches reinforced their role in glucose transport. In the present study, an attempt was made to identify the mechanism behind the glucose uptake activity of MFMC in in vitro using L6 myotubes.

In this context, the L6 muscle cell line was a suitable in vitro model to study glucose transport activity. Moreover, skeletal muscle is the major site for primary glucose clearance and glucose utilization ${ }^{[15]}$. Earlier reports of L6 myotubes evidenced the maximum glucose uptake in the presence of troglitazone and rosiglitazone at 10 and $100 \mu \mathrm{mol} \cdot \mathrm{L}^{-1}$ concentrations, respectively ${ }^{[8]}$. In addition, the enhanced glucose uptake in L6 cells is mostly mediated through elevated GLUT-4 levels ${ }^{[8,15]}$. In similar terms, the findings in the present study are in agreement with the literature reports where there is a concomitant increase in glucose uptake along with the enhanced GLUT-4 levels in L6 myotubes, the MFMC has shown enhanced glucose uptake, and assisted the enhanced GLUT-4 expression. Furthermore, cycloheximide, a protein synthesis inhibitor, has completely blocked troglitazone-mediated glucose uptake on chronic exposure, suggesting the importance of new protein synthesis for glucose transport ${ }^{[8]}$. Similarly, in the present study the glucose uptake activity of MFMC was completely abolished by cycloheximide, which clearly confirmed the role of new protein synthesis relevant to glucose transport.

Together, the above considerations established that glucose uptake is dependent on increased or decreased expression of GLUT-4; Moreover, the enhanced glucose uptake in the present study was concomitant with the increased expression of GLUT-4 encoding mRNA in L6 myotubes upon incubation with MFMC ${ }^{[16]}$.

Additionally, the expression of PPAR $\gamma$ was also increased, together with increased expression of GLUT-4, and enhanced glucose uptake in L6 myotubes when incubated with MFMC. In support of these findings, PPAR $\gamma$ agonists are known to increase the GLUT- 4 transcription by acting through PPAR $\gamma$ receptors ${ }^{[17]}$.

Interestingly, the findings of the present study have convincing inference to understand the mechanism governing the activation of glucose transport associated with MFMC. All the findings in the present study are in agreement to support the glucose uptake enhancing property of MFMC. Based on the gene expression study outcomes it was hypothesized that methanolic fruit extracts of $M$. cymbalaria could activate the glucose transport by up-regulation of GLUT-4 and PPAR $\gamma$ by acting as PPAR $\gamma$ agonist. Duplication of the above study in 3T3 L1 adipocyte cell-line could help to better understand the mechanism of action of M. cymbalaria.

\section{Conclusion}

In the current study, the unifying approach of medicinal chemistry and in vitro screenings assays was manifested, which assured the evidence of a set of targets on glucose transport. Also this study demonstrated the importance of GLUT-4, and PPAR $\gamma$ up-regulation by the methanolic fruit extract of $M$. cymbalaria in enhancing glucose transport. Its role in augmenting glucose uptake by modulating a battery of targets like GLUT-4, PPAR $\gamma$ and their significance in such processes has been studied. Purification of the above plant extract towards the isolation of the active principle(s) is in progress.

\section{Acknowledgement}

The authors greatly acknowledge Dr. Sandeep Varma, The Himalaya Drug Company, Bangalore for his valuable technical support. The authors also acknowledge Padmashree Institute of Information Sciences, Bangalore for providing funds for this research.

\section{References}

[1] Kameshwara Rao B, Keshavalu MM, Giri R, et al. Antidiabetic and hypolipidemic effects of Momordica cymbalaria Hook fruit powder in alloxan diabetic rats [J]. J Ethnopharmacol, 1999, 67 (1): 103-109.

[2] Kameshwara Rao B, Keshavalu MM, Apparao CH. Evaluation of antidiabetic effect of Momordica cymbalaria fruit in alloxandiabetic rats [J]. Fitoterapia, 2003, 74 (1-2): 7-13.

[3] Leung L, Birtwhistle R, Kotecha J, et al. Anti-diabetic and hypoglycaemic effects of Momordica charantia (bitter melon): a mini review [J]. Br J Nutr, 2009, 102 (12): 1703-1708.

[4] Raju BK, Suman S, Channakeshava TR. Antidiabetic activity of a tritepenoid saponin isolated from Momordica cymbalaria [J]. In J Exp Biol, 2014, 52 (1): 46-52.

[5] Ziel, FH, Venkatesan N, Davidson MB. Glucose transport is rate limiting for skeletal muscle glucose metabolism in normal and STZ-induced diabetic rats [J]. Diabetes, 1988, 37 (7): 885-890.

[6] Fukumoto H, Kayano T, Buse JB, et al. Cloning and characterization of the major insulin responsive glucose transporter expressed in human skeletal muscle and other insulin responsive tissues [J]. J Biol Chem, 1989, 264 (14): 7776-7779.

[7] Kellerer M, Lammers R, Haring HU. Insulin signal transduction; possible mechanism for insulin resistance [J]. Exp Clin Endocrinol Diabetes, 1999, 107 (2): 97-106.

[8] Ciaraldi TP, Huber KK, Hickman M, et al. Regulation of glucose transport in cultured muscle cells by novel hypoglycemic agents [J]. Metabolism, 1995, 44 (8): 976-981.

[9] Desvergne B, Wahli W. Peroxisome proliferator activated receptors: nuclear control of metabolism [J]. Endocrine Rev, 1999, 20 (5): 649-688.

[10] Ntambi JM, Young CK. Adipocyte differentiation and gene expression [J]. J Nutr, 2000, 130 (12): 3122S-3126S.

[11] Hall LR, Mehlotra RK, Higgins AW, et al. An essential role for interleukin-5 and eosinophils in helminth-induced airway hyper-responsiveness [J]. Infect Immun, 1998, 66 (9): 
4425-4430.

[12] Yonemitsu S, Nishimura H, Shintani M, et al. Troglitazone induces GLUT-4 translocation in L6 myotubes [J]. Diabetes 2001, 50 (5): 1093-1101

[13] Laville M, Auboeuf D, Khalfallah Y, et al. Acute regulation by insulin of phosphatidylinositol- 3-kinase, Rad, GLUT 4, and lipoprotein lipase mRNA levels in human muscle [J]. J Clin Invest, 1996, 98 (1): 43-49.

[14] Petersen KF, Krssak M, Inzucchi S, et al. Mechanism of troglitazone action in type 2 diabetes [J]. Diabetes 2000, 49 (5): 827-831.

[15] Koivisto UM, Martinez VH, Bilan PJ, et al. Differential regula- tion of the GLUT1 and GLUT4 glucose transport systems by glucose and insulin in L6 muscle cells in culture [J]. $J$ Biol Chem, 1991, 266 (4): 615-2621.

[16] Garvey WT, Maianu L, Zhu JH. Multiple defects in the adipocyte glucose transport system cause cellular insulin resistance in gestational diabetes. Heterogeneity in the number and a novel abnormality in subcellular localization of GLUT4 glucose transporters [J]. Diabetes, 1993, 42 (12): 1773-1785.

[17] Shimaya A, Kurosaki E, Shioduka K, et al. YM268 increases the glucose uptake, cell differentiation, and mRNA expression of glucose transporter in 3T3-L1 adipocytes [J]. Hormone Metab Res, 1998, 30 (9): 543-548.

Cite this article as: Puttanarasaiah Mahesh Kumar, Marikunte V Venkataranganna, Kirangadur Manjunath, Gollapalle L Viswanatha, Godavarthi Ashok. Methanolic extract of Momordica cymbalaria enhances glucose uptake in L6 myotubes in vitro by up-regulating PPAR- $\gamma$ and GLUT-4[J]. Chinese Journal of Natural Medicines, 2014, 12(12): 895-900 